\title{
Conidial fungi from the semi-arid Caatinga biome of Brazil. Ellisembiopsis gen. nov., new variety of Sporidesmiella and some notes on Sporidesmium complex
}

\section{Santa Izabel TS ${ }^{1 *}$, Cruz ACR $^{1}$ and Gusmão LFP ${ }^{1}$}

${ }^{1}$ Universidade Estadual de Feira de Santana. Av. Transnordestina, S/N - Novo Horizonte, 44036-900. Feira de Santana, BA, Brazil.tazuefsbot@gmail.com

Santa Izabel TS, Cruz ACR, Gusmão LFP 2013 - Conidial fungi from the semi-arid Caatinga biome of Brazil. Ellisembiopsis gen. nov., new variety of Sporidesmiella and some notes of Sporidesmium complex. Mycosphere 4(2), 156-163, Doi 10.5943/mycosphere/4/2/1

During an inventory of conidial fungi from dead plant material in the semi-arid Caatinga biome of Brazil, some species of Sporidesmium complex were found. Ellisembiopsis brasiliensis gen. et sp. nov. and Sporidesmiella garciniae var. brasiliensis var. nov. are proposed, described and illustrated. Sporidesmium knawiae is considered synonymous with Repetophragma inflatum. Eight new combinations of Ellisembia (E. delavayae, E. filirostrata, E. ilicis, E. magnibrachypa, E. ochnae, E. phoebes, E. pruni, E. tarennae) and, Ellisembiopsis zhejiangensis comb. nov. are proposed. A table comparing the main features of Sporidesmium and related genera is included.

Key words - Ellisembia - Ellisembiopsis - Sporidemiella - Sporidesmiopsis

\section{Article Information}

Received 20 December 2012

Accepted 10 January 2013

Published online 13 March 2013

*Corresponding author: Tasciano Santa Izabel - e-mail - tazuefsbot@ gmail.com

\section{Introduction}

The genus Sporidesmium Link was reviewed by Ellis (1958). At this time, the genus circumscription included species that have conidiophores solitary or in groups, conidiogenous cells with or without proliferation and smooth or verrucose, euseptate or distoseptate conidia. These characteristics could be segregated in different genera and/or subgenera, and were considered as Sporidesmium complex or Sporidesmium sensu lato (Sutton \& Hodges 1978, Hughes 1979).

Delimitation of the Sporidesmium complex was initiated when Sporidesmiella P.M. Kirk was erected to included species that have cylindrical to cuneiform or obovate and distoseptate conidia (Kirk 1982). Later, Sporidesmiopsis Subram. \& Bhat was proposed to accommodate the species with apically branched conidiophores (Subramanian \& Bhat 1987).

Subramanian (1992) divided the Sporidesmium complex into seven genera, based on conidia septation, proliferation of conidiogenous cells and presence or absence of conidiophores. Thus, he proposed a new circumscription for Sporidesmium and expanded the concept of Sporidesmiella. Sporidesmium was restricted to those species that have conidiogenous cells without or with irregularly proliferation, and euseptate conidia those species with annellidic proliferation and 
distoseptate conidia were included in Sporidesmiella.

Hernández-Gutierrez \& Sutton (1997) proposed two new genera. Imimyces A. Hern. Gut. \& B. Sutton for those species with conidiophores associated with lageniform, doliiform or ovoid conidiogenous cells, with percurrent proliferation and distoseptate conidia, and Linkosia A. Hern. Gut. \& B. Sutton for species with ampulliform conidiogenous cells, arising directly from the mycelium and producing distoseptate conidia. Imimyces densus (Sacc. \& Roum.) A. Hern. Gut. \& B. Sutton was typified based on Helminthosporium densum Sacc. \& Roum. (Hernández-Gutierrez \& Sutton 1997). However, they probably not studied the type of this species, but a part of the type species of $H$. leptosporum Sacc. \& Roum., considered by Ellis (1958) a synonym of $H$. densum (Shoemaker \& Hambleton 2001). Hughes (1958) analyzed type material of $H$. densum and $H$. leptosporum considered the first one, synonymous to Polydesmus elegans Durieu \& Mont. and the last one should not be a synonym of $H$. densum. Thus, Shoemaker \& Hambleton (2001) proposed the exclusion of Imimyces and proposed a new genus Imicles Shoemaker \& Hambl. with type species Imicles leptospora (Sacc. \& Roum.) Shoemaker \& Hambl. to accommodate the species previously included in Imimyces.

According to $\mathrm{Wu} \&$ Zhuang (2005), species delimitation of SporidesmiumPenzigomyces and Ellisembia-Imicles remain tenuous, and they suggested that the species included in Penzigomyces Subram. and Imicles should be transferred to Sporidesmium and Ellisembia respectively.

This paper adopts Subramanian (1992) and the concept of Polydesmus proposed by Hernández-Gutierrez \& Sutton (1997).

In the last decade, the number of species in the Sporidesmium complex has increased (Shi \& Zhang 2007, Ma \& Zhang 2007ab, Zhang et al. 2008, Ma et al. 2010). Nevertheless, important features such as the conidium septation and conidiophores reduced to conidiogenous cells, have been questioned (Hughes \& Illman 1974, Réblová 1999).

Castañeda-Ruiz et al. (2011) proposed a new species and re-disposed taxa in
Repetophragma in accordance with Subramanian (1992). According to this author, macronematous conidiophores with several annellations produced by percurrent proliferations of the conidiogenous cell and euseptate conidia are the diagnostic characters of Repetophragma.

A phylogenetic analysis based on partial sequences of LSU nu-rDNA and RPB2 of 27 species and nine genera of the Sporidesmium complex, demonstrated multiple evolutionary origins and distributed phylogenetically in Dothideomycetes and Sordariomycetes (Shenoy et al. 2006).

Despite this, Shenoy et al. (2006) favoured maintenance of the genera in the Sporidesmium complex, until the sexual phase of these anamorphic fungi are found.

Further studies are needed, with greater sampling effort and the use of more genetic markers for the genera/species to elucidate this complex.

\section{Methods}

Expeditions were conducted from 2008 to 2009 in semi-arid Caatinga biome, Northeast of Brazil. Samples of plant litter were collected in separate paper bags and taken to the laboratory where each was incubated at $25^{\circ} \mathrm{C}$ in Petri dish 'moist chambers'. The plant material was screened at regular intervals for microfungi. Mounts were prepared in polyvinyl alcohol-glycerol (8 g per $100 \mathrm{ml} \mathrm{H}_{2} 0,5 \mathrm{ml}$ glycerol). Specimens are deposited in the Herbarium of Universidade Estadual de Feira de Santana (HUEFS).

\section{Results}

Ellisembiopsis T.S. Santa Izabel \& Gusmão gen. nov.

MycoBank 803131

Subram.

Etymology - related to Ellisembia

Dematiceous hyphomycetes. Conidiophores macronematous, mononematous, erect, straight or flexuous, branched at the apex, septate, dark brown; conidiogenous cells monoblastic, integrated, lageniform to doliform; conidia solitary, obclavate to fusiform, distoseptate. Secession schizolytic. 
Type species - Ellisembiopsis brasiliensis T.S. Santa Izabel \& Gusmão.

Notes - The genus Ellisembiopsis is similar to Ellisembia and Sporidesmiopsis. It differs from Ellisembia by apical branched conidiophores and differing from Sporidesmiopsis by distoseptate conidia.

Ellisembiopsis brasiliensis T.S. Santa Izabel \& Gusmão sp. nov.

Fig. 1

MycoBank 803132

Etymology - named for the country where it was collected.

Conidiophores macronematous, mononematous, erect, straight or flexuous, branched at the apex region, 13-15-septate, smooth, dark brown, 270-462 × 16.5-20 $\mu \mathrm{m}$; apex, 4-7.5 wide; conidiogenous cells monoblastic, integrated, lageniform to doliform, 0-3 proliferations, $8-13 \times 4-6 \mu \mathrm{m}$; conidia solitary, obclavate to fusiform 5-9-distoseptate, smooth, central cells dark brown, polar cells light brown, 35-80 × 7.5-13.5 $\mu \mathrm{m}, 3.5-6 \mu \mathrm{m}$ wide at the base, $2.5-4 \mu \mathrm{m}$ wide at the apex. Secession schizolytic. substrate.

Teleomorph - not observed on natural

Know distribution - Brazil.

Material examined - Brazil, Bahia, Morro do Chapéu, on decaying twig, 23 Dec 2008, T.S. Santa Izabel. HUEFS 155102 (holotype). Ibid. on decaying twig, 06 Oct 2008, T.S. Santa Izabel. HUEFS 155101.

Notes - Ellisembiopsis brasiliensis is similar to Sporidesmiopsis zhejiangensis by possessing distoseptate conidia, however, the latter species has verruculose and smaller conidia with fewer distosepta (5-7) and presence of mucilage at the apex (Wongsawas et al. 2008). Sporidesmiopsis goanensis Bhat \& Kendr. and S. dennisii (Crane \& Dumont) Bhat, Kendr. \& Nag Raj have 3-4 and 10-15euseptate conidia, respectively (Bhat \& Kendrick 1993, Subramanian \& Bhat 1987).

Ellisembiopsis zhejiangensis (Wongsawas, H.K. Wang, K.D. Hyde \& F.C. Lin., J.) T.S. Santa Izabel \& Gusmão comb. nov.

MycoBank 803134

Bas.: Sporidesmiopsis zhejiangensis Wongsawas, H.K. Wang, K.D. Hyde \& F.C. Lin., J. Zhejiang, Univ., Sci. B 9: 798. 2008.
Sporidesmiella garciniae var. brasiliensis T.S. Santa Izabel \& Gusmão var. nov. $\quad$ Fig. 2 MycoBank 803133

Etymology - named for the country where it was collected.

Conidiophores macronematous, mononematous, solitary or in groups, erect, straight or flexuous, unbranched, smooth, brown, subhyaline at the apex, 32-106 × 3.5$5.5 \mu \mathrm{m}$; conidiogenous cells polyblastic, integrated, terminal, cylindrical, smooth, sympodial proliferations or with percurrent and sympodial proliferations; conidia solitary, dry, cylindrical to subcylindrical, smooth, 4distoseptate, light brown to olive grey, base subhyaline, $22.5-28.5 \times 2.5-4.0 \mu \mathrm{m}$. Secession schizolytic.

Teleomorph - not observed on natural substrate.

Know distribution - Brazil.

Material examined - Brazil, Bahia, Morro do Chapéu, on dead leaf of an unidentified dicotyledonous plant, 23 May 2008, T.S. Santa Izabel. HUEFS 155106. (holotype).

Notes - The genus Sporidesmiella P.M. Kirk comprises 24 species and four varieties (Yanna et al. 2001, Wu \& Zhuang 2005). Sporidesmiella hyalosperma var. novaezelandiae (S. Hughes) P.M. Kirk and Sporidesmiella parva var. palauensis Matsush. were proposed by presence of conidiogenous cell with sympodial proliferation, differing from conidiogenous cell with percurrent proliferations commonly present in the genus (Kirk 1982, Matsushima 1985). Sporidesmiella garciniae Matsush. var. garciniae has larger conidiophores than $S$. garciniae var. brasiliensis and has conidiogenous cells only with percurrent proliferations (Matsushima 1985).

Notes - According to Subramanian (1992) Sporidesmium and Ellisembia differ by possessing euseptate and distoseptate conidia, respectively (Table 1). According to these characteristics and based on original descriptions and illustrations, eight new combinations are proposed for Ellisembia (Shi \& Zhang 2007, Ma \& Zhang 2007a).

Ellisembia delavayae (Cheng K. Shi \& X.G. Zhang) T.S. Santa Izabel, A.C. Cruz \& 


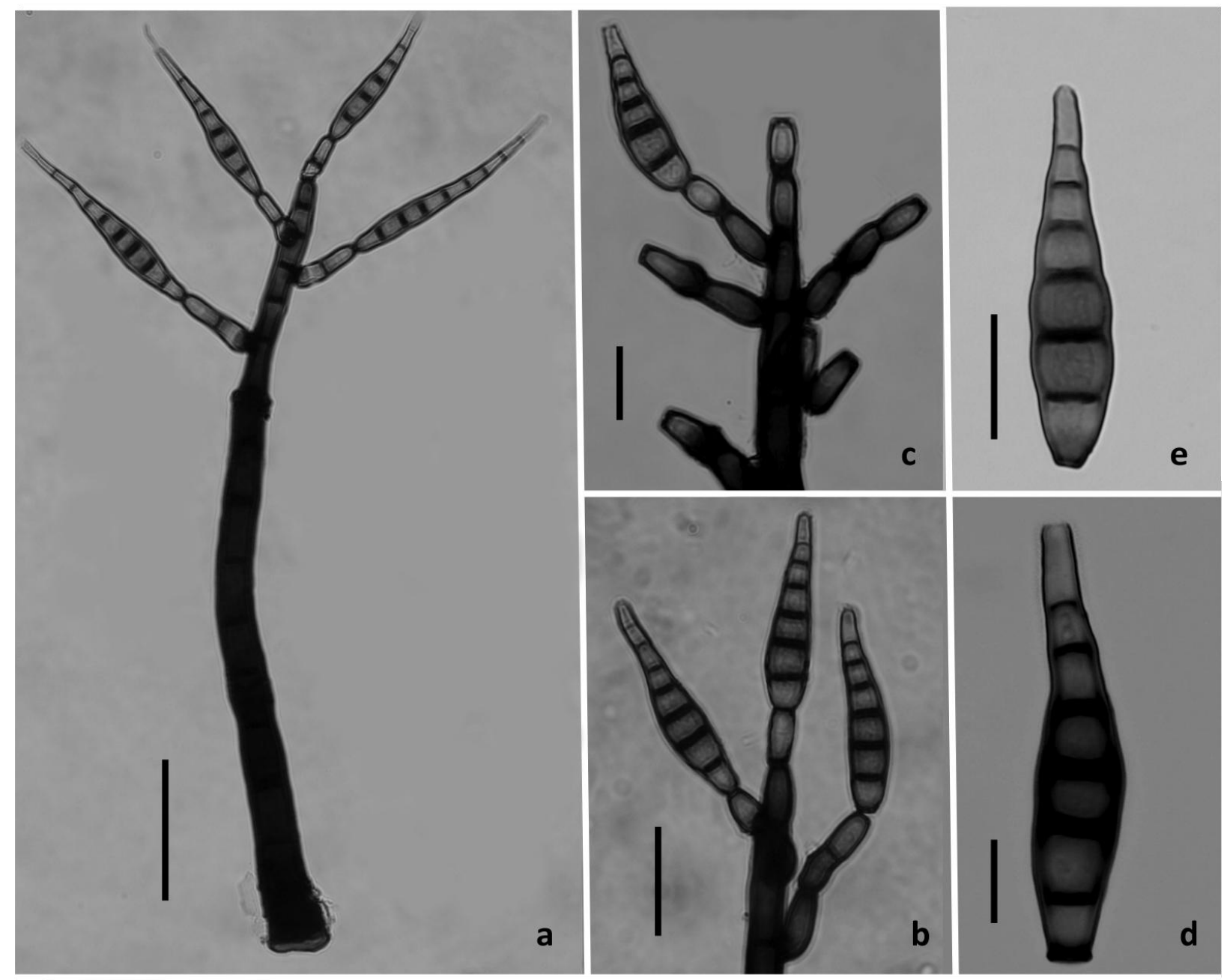

Fig. 1 - a-e Ellisembiopsis brasiliensis. a General aspect. b-c Details of branches of conidiophores and conidiogenous cells. d-e Conidia. Bars $=10 \mu \mathrm{m}(\mathrm{d}) ; 20 \mu \mathrm{m}$ (b, c, e) $50 \mu \mathrm{m}$ (a).

Gusmão comb. nov.

MycoBank 803135

Bas.: Sporidesmium delavayae Cheng K. Shi \& X.G. Zhang, Mycotaxon 99: 359. 2007.

Ellisembia filirostrata (Cabello, Cazau \& Aramb.) T.S. Santa Izabel, A.C. Cruz \& Gusmão comb. nov.

MycoBank 803136

Bas.: Sporidesmium filirostratum Cabello, Cazau \& Aramb., Mycotaxon 38: 16. 1990.

Ellisembia ilicis (Jian Ma \& X.G. Zhang) T.S. Santa Izabel, A.C. Cruz \& Gusmão comb. nov. MycoBank 803137

Bas.: Sporidesmium ilicis Jian Ma \& X.G. Zhang, Mycotaxon 99: 369. 2007.
Ellisembia magnibrachypa (Matsush.) T.S. Santa Izabel, A.C. Cruz \& Gusmão comb. nov. MycoBank 803138

\section{Bas.: Sporidesmium magnibrachypus}

Matsush., Icones Microfungorum a Matsushima lectorum: 138. 1975.

Ellisembia ochnae (Cheng K. Shi \& X.G. Zhang) T.S. Santa Izabel, A.C. Cruz \& Gusmão comb. nov.

MycoBank 803139

Bas.: Sporidesmium ochnae Cheng K. Shi \& X.G. Zhang, Mycotaxon 99: 365. 2007.

Ellisembia phoebes (Cheng K. Shi \& X.G. Zhang) T.S. Santa Izabel, A.C. Cruz \& Gusmão comb. nov.

MycoBank 803140

Bas.: Sporidesmium phoebes Cheng K. Shi \& X.G. Zhang, Mycotaxon 99: 362. 2007. 


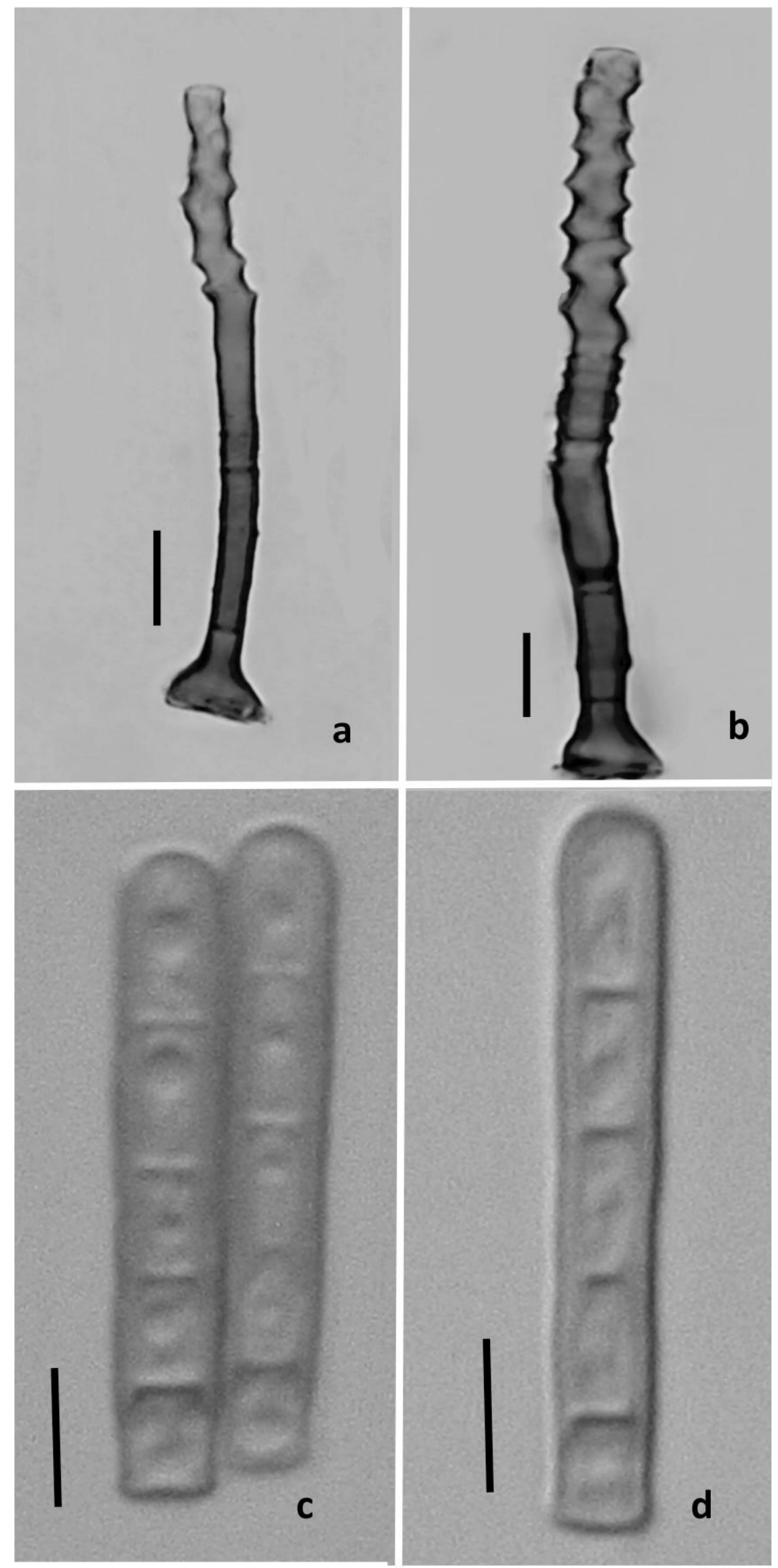

Fig. 2 - Sporidesmiella garciniae var. brasiliensis. a Conidiophores and conidiogenous cells with sympodial proliferations. b Conidiophores and conidiogenous cells with sympodial and percurrent proliferations. $\mathbf{c}-\mathbf{d}$. Conidia. Bars $=5 \mu \mathrm{m}(\mathrm{c}, \mathrm{d}) 10 \mu \mathrm{m}(\mathrm{a}, \mathrm{b})$. 
Ellisembia pruni (Jian Ma \& X.G. Zhang) T.S. Santa Izabel, A.C. Cruz \& Gusmão comb. nov. MycoBank 803141

Bas.: Sporidesmium pruni Jian Ma \& X.G. Zhang, Mycotaxon 99: 369. 2007.

Ellisembia tarennae (Cheng K. Shi \& X.G. Zhang) T.S. Santa Izabel, A.C. Cruz \& Gusmão comb. nov.

MycoBank 803142

Bas.: Sporidesmium tarennae Cheng K. Shi \& X.G. Zhang, Mycotaxon 99: 361. 2007.

Repetophragma inflatum (Berk. \& Ravenel) W.P. Wu, Fung. Divers. Res. Ser. 15: 109, 2005.

Bas.: Helminthosporium inflatum Berk. \& Ravenel, Grevillea 3: 104. 1875.

Syn.: Helminthosporium subfuscum Berk. \& M.A. Curtis, Grevillea 3: 104. 1875.

Helminthosporium tiara Berk. \& Ravenel, Grevillea 3(27): 104. 1875.

Helminthosporium episphaericum

Cooke \& Peck, Rep. N.Y. St. Mus. nat. Hist. 29: 52.1878.

Helminthosporium collabendum Cooke, Grevillea 17: 67. 1889.

Clasterosporium herculeum Ellis, Proc. Acad. nat. Sci. Philad.: 92. 1891.

Clasterosporium sigmoideum Ellis \&

Everh., Bull. Torrey bot. Club 24: 472. 1897.

Sporidesmium inflatum (Berk. \&

Ravenel) M.B. Ellis, Mycol. Pap. 70: 70.1958.

Sporidesmium knawiae Crous, Fungal

Planet 29. 2008. syn. nov.

Material examined - Brazil, Piauí, Caracol, on decaying twig, 5 Dec 2006. ACR Cruz, HUEFS 130991.

Note - Sporidesmium knawiae proposed by Crous (2008) is phylogenetically and morphologically similar to Repetophragma inflatum. The characteristics of this species are fully included in the morphological variation of the species previously described by Ellis (1958). Thus, it is proposed to make Sporidesmium knawiae a synonym of Repetophragma inflatum.

\section{Other species of Sporidesmium complex found in semi-arid region}

Ellisembia adscendens (Berk.) Subram., Proc. Indian natn Sci. Acad., Part B. Biol. Sci. 58(4): 183. 1992.

Material examined - Brazil, Bahia, Morro do Chapéu, on dead bark, 19 May 2008, T.S. Santa Izabel. HUEFS 155127. 21 Oct. 2008, T.S. Santa Izabel. HUEFS 155128, 9 Jan 2009, T.S. Santa Izabel. HUEFS 155129; on decaying twig, 23 May 2008, T.S. Santa Izabel. HUEFS 155130, 7 Oct 2008, T.S. Santa Izabel. HUEFS 155131, 31 Jan 2008, T.S. Santa Izabel. HUEFS 155132.

Ellisembia brachypus (Ellis \& Everh.)

Subram., Proc. Indian natn Sci. Acad., Part B. Biol. Sci. 58: 183. 1992.

Material examined - Brazil, Bahia, Morro do Chapéu, on decaying twig, 2 Oct 2008, T.S. Santa Izabel. HUEFS 155133.

Ellisembia vaga (Nees \& T. Nees) Subram., Proc. Indian Natn. Sci. Acad., Part B. Biol. Sci. 58(4): 184. 1992.

Material examined - Brazil, Bahia, Morro do Chapéu, on decaying twig, $20 \mathrm{Jul}$ 2008, T.S. Santa Izabel, HUEFS 155104.

Sporidesmiella aspera Kuthub. \& Nawawi, Mycol. Res. 97(11): 1305.1993.

Material examined - Brazil, Bahia, Morro do Chapéu, on dead leaves, 24 Oct 2008, T.S. Santa Izabel. HUEFS 155136; on dead bark, 23 May 2008, T.S. Santa Izabel,HUEFS 155137; 17 Feb 2008, T.S. Santa Izabel, HUEFS 155138; on decaying twig, 23 Dec 2008, T.S. Santa Izabel. HUEFS 155139 .

Sporidesmiella cuneiformis (B. Sutton) P.M. Kirk, Trans. Br. mycol. Soc. 79(3): 481. 1982.

Material examined - Brazil, Bahia, Morro do Chapéu, on decaying twig, 11 Jun 2008, T.S. Santa Izabel. HUEFS 155140. 
Table 1 Main features of Sporidesmium complex and related genera.

\begin{tabular}{|c|c|c|c|c|}
\hline Genera & Condiophores & Conidiogenous cells & Conidia & \\
\hline Ellisembia & Present & Irregular proliferations & $\begin{array}{l}\text { Distoseptate/unbranched/ } \\
\text { appendage }\end{array}$ & \\
\hline Ellisembiopsis & Present/Branched & Percurrent proliferations & Distoseptate & \\
\hline Imicles & Present & Percurrent proliferations & $\begin{array}{l}\text { Distoseptate/unbranched/ } \\
\text { appendage }\end{array}$ & \\
\hline Janetia & Absent & Without proliferations & Euseptate/Distoseptate & \\
\hline Linkosia & Absent & Without proliferations & Distoseptate & \\
\hline Lomaantha & Present & Without proliferations & Distoseptate/branched & \\
\hline Morrisiella & Synnematous & Without proliferations & Distoseptate & \\
\hline Neosporidesmium & Synnematous & Precurrent proliferations & $\begin{array}{l}\text { Distoseptate/unbranched/ } \\
\text { appendage }\end{array}$ & \\
\hline Novozymia & Synnematous & Annelidic proliferations & $\begin{array}{l}\text { Distoseptate/unbranched/ } \\
\text { appendage }\end{array}$ & \\
\hline Penzigomyces & Present & Percurrent proliferations & $\begin{array}{l}\text { Euseptate/unbranched/ } \\
\text { appendage }\end{array}$ & \\
\hline Polydesmus & Present & Without proliferations & Euseptate & \\
\hline Repetophragma & Present & Annelidic proliferations & Euseptate & \\
\hline Sporidesmiella & Present & Annelidic proliferations & $\begin{array}{l}\text { Distoseptate/unbranched } \\
\text { branched/ appendage }\end{array}$ & or \\
\hline Sporidesmina & Synnematous & Without proliferations & Euseptate/Distoseptate & \\
\hline Sporidesmiopsis & Present/Branched & Percurrent proliferations & Euseptate & \\
\hline Stanjehughesia & Absent & Without proliferations & Euseptate & \\
\hline
\end{tabular}

Sporidesmium tropicale var. tropicale M.B. Ellis, Mycol. Pap. 70: 58. 1958.

Material examined - Brazil, Bahia, Morro do Chapéu, on dead bark, 13 Feb 2008, T.S. Santa Izabel. HUEFS 155141.

\section{Acknowledgements}

The authors thank the "Programa de Pós-graduação em Botânica - PPGBot/UEFS" and CNPq. Part of the support for this work came from the Program of Research of Biodiversity in the Brazilian Semi-arid (PPBIO Semi-arid/Ministry of Technology and Science - proc. 558317/2009-0)

\section{References}

Bhat DJ, Kendrick WB. 1993. Twenty-five new conidial fungi from the Western Ghats and the Andaman Islands (India). Mycotaxon 49, 19-90.

Castañeda-Ruiz RF, Heredia G, Arias RM, McKenzie EHC, Hyde KD, Stadler M, Saikawa M, Gené J, Guarro J, Iturriaga T, Minter DW \& Crous PW. 2011. A new species and re-disposed taxa in Repetophragma. Mycosphere 2(3), 273289.
Crous PW. 2008. Sporidesmium knawiae Crous, sp. nov. Fungal Planet 29.

Ellis MB. 1958. Clasterosporium and some allied dematiaceae-phragmosporae. I. Mycological Papers 70, 1-89.

Hernández-Gutiérrez A, Sutton BC. 1997. Imimyces and Linkosia, two new genera segregated from Sporidesmium sensu lato, and redescription of Polydesmus. Mycological Resarch 101, 201-209.

Hughes SJ. 1958. Revisiones Hyphomycetum aliquot cum appendice de nominibus rejiciendis. Canadian Journal of Botany 36, 727-836.

Hughes SJ, IllmanWI. 1974. Sporidesmium larvatum. Fungi Canadenses 58, 1-2.

Hughes, SJ. 1979. Relocation of species of Endophragmia auct. with notes on relevant generic names. New Zealand Journal of Botany 17, 139-188.

Kirk PM. 1982. New or interesting microfungi VI. Sporidesmiella gen. nov. (Hyphomycetes). Transactions of the British Mycological Society 79, 479489.

Ma J, Zhang XG. 2007a. Taxonomic studies of Sporidesmium from China. Mycotaxon 99, 367-372. 
Ma J, Zhang XG. 2007b. Two new species of Sporidesmium from Yunnan, China. Mycotaxon 101, 73-76.

Ma, J, Zhang, YD, Ma, LG, Ren, SC, Zhang, XG. 2010. Taxonomic studies of Ellisembia from Hainan, China. Mycotaxon 114, 417-421.

Matsushima T. 1985. Matsushima Mycological Memoirs no. 4. Kobe. Published by the author.

Réblová M. 1999. Studies in Chaetosphaeria sensu lato III. Umbrinosphaeria gen. nov. and Miyoshiella with Sporidesmium anamorphs. Mycotaxon 71, 13-43.

Shenoy BD, Jeewon R, Wu WP, Bhat DJ, Hyde KD. 2006. Ribosomal and RPB2 DNA sequence analyses suggest that Sporidesmium and morphologically similar genera are polyphyletic. Mycological Research 110, 916-928.

Shi CK, Zhang XG. 2007. Taxonomic studies of Sporidesmium from Guangxi, China. Mycotaxon 99, 359-366.

Shoemaker RA., Hambleton S. 2001. "Helminthosporium" asterinum, Polydesmus elegans, Imimyces, and allies. Canadian Journal of Botany 79, 592-599.

Subramanian CV. 1992. A reassessment of Sporidesmium (Hyphomycetes) and some related taxa. Proceedings of the Indian National Sciences Academy 58, 179190.

Subramanian CV, Bhat DJ. 1987. Hyphomycetes from South India. I. Some new taxa. Kavaka 15(1-2), 41-74.

Sutton BC, Hodges CS. 1978. Eucalyptus microfungi. Chaetendophragmiopsis gen. nov. and other Hyphomycetes. Nova Hedwigia 29, 593-607.

Wongsawas M, Wang HK, Hyde KD, Lin, FC. 2008. New and rare lignicolous hyphomycetes from Zhejiang Province, China. Journal of Zhejiang University Science B. 9(10), 797-801.

Wu W, Zhuang W. 2005. Sporidesmium, Endophragmiella and related genera from China. Fungal Diversity Research Series 15. Hong Kong, Fungal Diversity Press.

Yanna, Ho WH, Hyde KD. 2001. Sporidesmiella oraniopsis, a new species of dematiaceous hyphomycetes from North Queensland, Australia and synopsis of the genus. Fungal Diversity 8, 183-190.

Zhang K, Ma J, Zhang XG. 2008. Taxonomic studies of Sporidesmium from Hainan, China. Mycotaxon 104, 165-169. 